\title{
Hyaluronan-mediated ferric oxide nanoparticles causes apoptosis of CD44 expressing head and neck squamous cell carcinoma cells
}

\author{
Ranjeeta Thapa',5, Jason Gorski², Anthony Bogedin'2, Michael Maywood ${ }^{3}$, Christopher Clement', \\ Seyedmehdi Hossaini Nasr ${ }^{4}$, Darrin Hanna ${ }^{2}$, Xuefei Huang ${ }^{4}$, Bradley J Roth ${ }^{5}$, \\ Gerard Madlambayan ${ }^{3}$, George D Wilson ${ }^{1}$
}

\author{
${ }^{1}$ Department of Radiation Oncology, William Beaumont Hospital, Royal Oak, USA \\ ${ }^{2}$ Department Electrical Engineering, Oakland University, Rochester, USA \\ ${ }^{3}$ Department of Biological Sciences, Oakland University, Rochester, USA \\ ${ }^{4}$ Department of Chemistry, Michigan State University, Lansing, USA \\ ${ }^{5}$ Department of Physics, Oakland University, Rochester, USA
}

Received January 29, 2016; Revised April 07, 2016; Accepted April 10, 2016; Published Online April 27, 2016

\section{Original Article}

\begin{abstract}
Purpose: To eliminate CD44, a putative cancer stem cell (CSC) marker, overexpressing head and neck squamous cell carcinoma (HNSCC) cells by using hyaluronan-conjugated, dextran-coated super paramagnetic iron oxide nanoparticles (HA-DESPIONs), in conjunction with induced heat produced by exposure to an alternating magnetic field (AMF). Methods: An AMF generator was constructed by means of a solenoid coil and an impedance circuit driven by a power amplifier. A signal generator produced a small sinusoidal signal of $130 \mathrm{kHz}$ that was then amplified to $9 \mathrm{~A}$ (peak to peak value) to generate an AMF of approximately 10 $\mathrm{kA} / \mathrm{m}$ (12.6 $\mathrm{mT}$ ) at the center of a coil. The heat generating effect of the AMF generator was tested via several kinetic and dose-dependent bulk heating experiments by exposing readily available magnetic nanoparticles to AMF. For elimination of CD44 population, UT-SCC-14 cells were exposed to either targeted HA-DESPIONs or non-targeted DESPIONs at a concentration $200 \mu \mathrm{g} / \mathrm{ml}$ and exposed to AMF for 30 minutes. Cells were processed after 24 hours for flow cytometry based analysis of apoptosis. Results: Magnetic nanoparticles caused a concentration-dependent bulk heating effect in response to AMF resulting in a significant temperature rise. Following the exposure to AMF, non-conjugated DESPIONs were unable to induce targeted hyperthermia and hence had no effect on CD44 cell death in HNSCC cells. However, there was a significant cell death in the CD44 population treated with HA-DESPIONs and exposed to AMF. This effect was only obeserved when the magnetic field was turned on. Conclusion: Bulk heating experiments concluded that a simple AMF generator was able to activate magnetic nanoparticles and flow cytometry demonstrated that HA- DESPIONs were able to cause apoptosis in UT-SCC-14 cells that express CD44.This may be a promising strategy to specifically target cancer stem cells (CSCs) for the treatment of HNSCC.
\end{abstract}

Keywords: HNSCC; CD44; HA-DESPIONs; AMF Generator; Magnetic Hyperthermia

\section{Introduction}

Head and neck squamous cell carcinoma (HNSCC) is the most frequent malignant form of head and neck cancer originating from the mucosal lining of the upper aerodigestive tract. HNSCC is the sixth most common cancer worldwide with approximately 5,00,000 new cases each year. ${ }^{1}$ Despite advances in technologies such as surgery, radiation therapy and chemotherapy, it still has a poor prognosis and survival rates have not 
improved substantially in the last decade. ${ }^{2}$ Currently there is much interest in the existence and activity of cancer stem cells (CSCs) in HNSCC as a potential reason why conventional therapies fail to eradicate tumors. ${ }^{3}$ CSCs possess intrinsic survival mechanisms that may protect them from radiotherapy and chemotherapy. ${ }^{4}$ The major challenges for the recent innovations are to specifically target CSCs. CD44 is a surface adhesive single-chain glycoprotein and a high affinity binding partner for hyaluronic acid/hyaluronan (HA) ${ }^{5,6}$ and has been identified as a putative CSC biomarker in HNSCC 4 . HA is a naturally occurring, polyanionic, polysaccharide and a major component of the extracellular matrix that plays a significant role in a variety of cell signaling processes such as wound healing, cell differentiation, proliferation, and migration. ${ }^{7}$ Recent evidence has strengthened the potential role of CD44 in HNSCC CSCs and their influence on disease progression and treatment outcome by investigating the mechanism of HA-CD44 interaction in the context of cancer progression and metastasis. ${ }^{8}$

Recently, superparamagnetic iron oxide nanoparticles (SPIONs) have been used extensively for cancer imaging, drug delivery and therapy (including chemotherapy).9,10 There are several developing treatment strategies aimed at HNSCC in addition to surgery, chemotherapy and radiation including targeted therapies ${ }^{11}$, hyperthermia and thermoablation therapy. In addition to these, ablative or thermotherapy using SPIONS has been investigated for head and neck cancers as an alternative, minimally invasive and inexpensive treatment. ${ }^{11,12}$ One promising thermotherapy strategy is targeted magnetic-activated hyperthermia. Magnetic hyperthermia can be achieved by delivering magnetic nanoparticles to cancer cells/tissue and creating local heat in the presence of an externally applied alternating magnetic field (AMF), which results in thermo-ablation of the cancer cells/tissue. ${ }^{13}$ The AMF-induced heating of these magnetic nanoparticles is through Néel relaxation and /or Brownian motion. ${ }^{14}$ Magnetic alternating current-based hyperthermia therapy has been introduced in the treatment of head and neck cancer and lung cancer ${ }^{12,15}$ while magnetic nanoparticle-based nanotherapy has been investigated for the treatment of tumors of the central nervous system ${ }^{\mathbf{1 6}, 17}$. In one recent study, induced hyperthermia was achieved for the treatment of head and neck cancer using a mouse xenograft model of a human HNSCC cell line. ${ }^{12}$ However, it is yet to be determined whether targeted SPIONs functionalized with $\mathrm{HA}$ exhibit targeted magnetic hyperthermia. Targeted magnetic hyperthermia would overcome some of the limitations of the current hyperthermia protocols, including heat induced ablation to the normal surroundings. To address this limitation, we have taken the approach of targeting a ligand specific receptor (in this case CD44) by using hyaluronan-conjugated, dextran-coated super paramagnetic iron oxide nanoparticles (HA-DESPIONs).
We have recently demonstrated that HA-DESPIONs were non-toxic and, although they did not enhance radiation sensitivity, they increased the effect of local hyperthermia.18 Further, these nanoparticles have previously been shown to be detectable in tumors and atherosclerotic plaques using static magnetic fields to provide contrast for magnetic resonance imaging. ${ }^{10,20}$ In this study, HA-DESPIONs with a diameter of $103 \mathrm{~nm}$, in conjunction with induced heat produced by exposure to an AMF, were evaluated for their effects on the viability of CD44 expressing HNSCC cell line using an in vitro model. To achieve the AMF-induced hyperthermia, an in-house fabricated AMF generator was constructed. We hypothesized that HA immobilized magnetic nanoparticles (HA-DESPIONs) can selectively target CD44 populations through CD44 binding in contrast to unmodified magnetic nanoparticles (DESPIONs).

\section{Methods and Materials}

\subsection{Cell line}

The UT-SCC-14 cell line was obtained through collaboration with Dr. Reidar Grénman (University of Turku, Finland). Clinicopathological characteristics of UT- SCC- 14 cell line showed that it was developed from the second grade primary lesion of the tongue of a 25 years old male patient with TNM staging as T3N1M0. It was maintained at low passage numbers such that it retained the phenotypic and morphological characteristics similar to the primary tumor. Overexpression of CD44 in UT-SCC-14 cells was previously conformed via flow cytometric analysis. ${ }^{18,21}$ The doubling time of the cell line is approximately 48 hours and the plating efficiency is $0.15-0.20$. Cells were cultured in Dulbecco modified eagle medium supplemented with $10 \%$ fetal bovine serum and $2 \%$ penicillin-streptomycin, and maintained in an incubator at $37^{\circ} \mathrm{C}$ with $5 \% \mathrm{CO}_{2} / 95 \%$ air.

\subsection{DESPIONs and HA-DESPIONs synthesis}

DESPIONs were processed by the formation of water soluble nanoparticles through co-precipitation with $\mathrm{Fe}^{3+}$ and $\mathrm{Fe}^{2+}$ salts $\left(\mathrm{FeCl}_{3}\right.$ and $\left.\mathrm{FeCl}_{2}\right)$ in the presence of dextran $(16 \mathrm{kDa})$ as previously described. ${ }^{22}$ HA-DESPIONs were prepared as previously described $^{19,22}$ which involved the conjugation of low molecular weight $\mathrm{HA}$ polymer $(31 \mathrm{kDa})$ in the presence of 2-chloro-4,6-dimethoxy-1,3,5-triazine (CDMT) as the coupling agent ${ }^{19}$ (Figure 1). The nanoparticles have been thoroughly characterized by transmission electron microscopy, thermogravimetric analysis, elemental analysis, dynamic light scattering, and high-resolution magic angle spinning NMR. ${ }^{22,23}$ The HA-DESPIONs used in this study had hydrodynamic radius of $103 \mathrm{~nm}$ with a core size of around $6 \mathrm{~nm}$, and a zeta potential of $-13 \mathrm{mV}$, while DESPIONs were about $59.6 \mathrm{~nm}$ with a zeta potential of $-4 \mathrm{mV}$. The thermogravimetric analysis data showed that HA accounted for $43 \%$ weight of each HA-DESPION. ${ }^{24}$ 


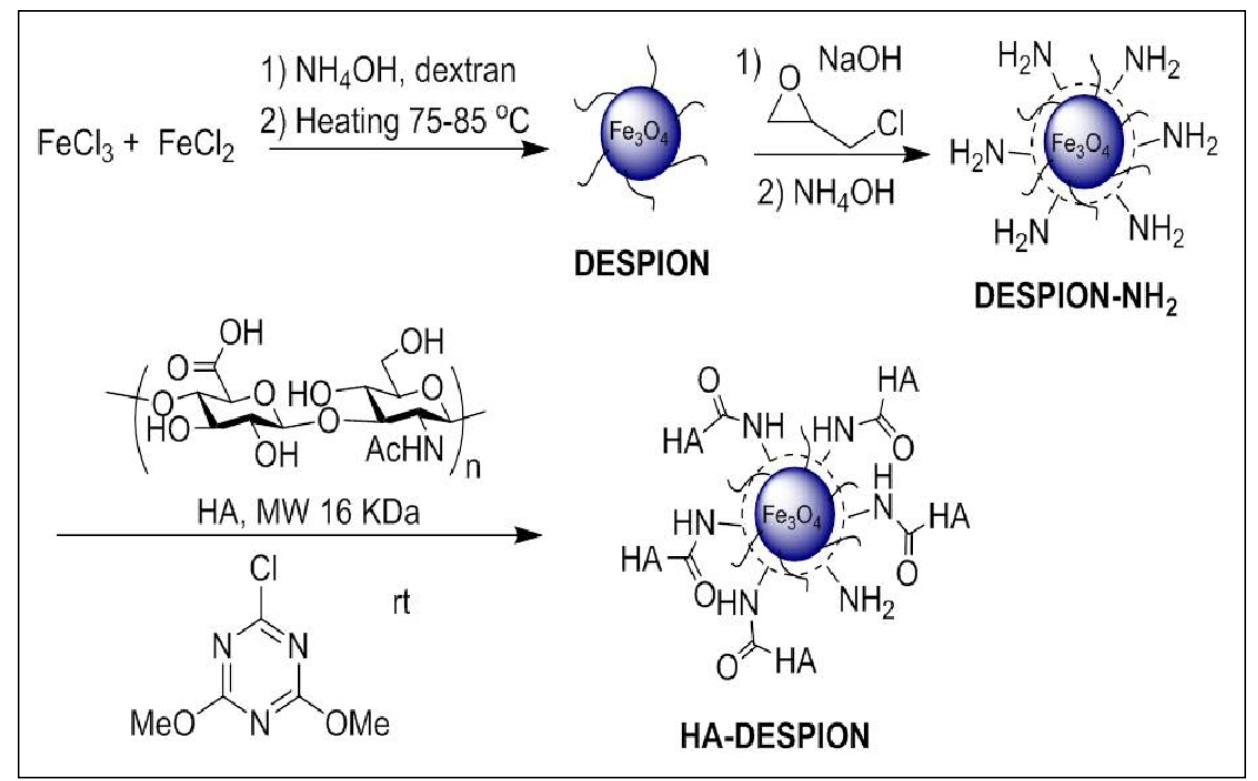

Figure 1: Synthesis of HA-DESPIONs. ${ }^{22}$

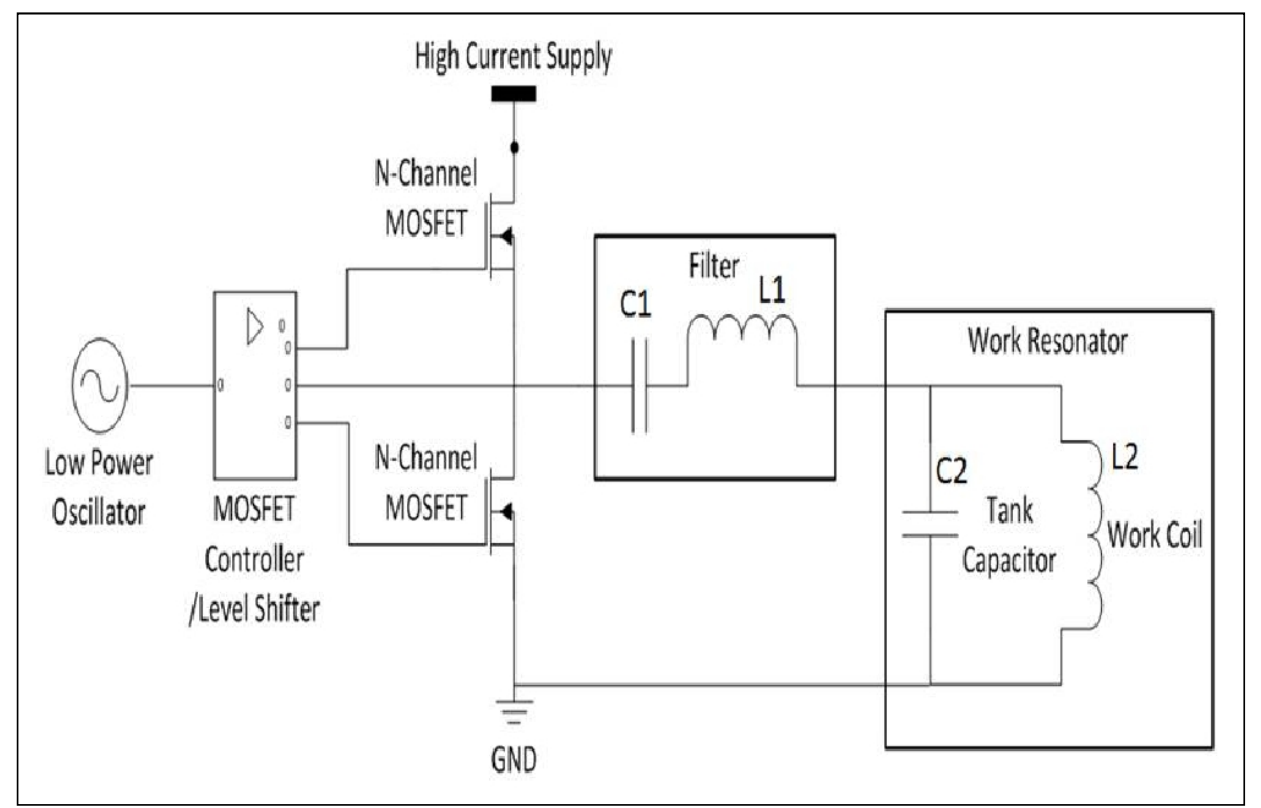

Figure 2: The circuit diagram of the AMF generator.

\subsection{Construction details of the AMF generator}

An AMF generator was constructed to generate AMF of high strength, thus allowing for a non-contact transfer of energy to a load. The generator consisted of a low power oscillator, a metal-oxide semiconductor field-effect transistor (MOSFET) amplifier, a filter and the work resonator. The low power oscillator was a programmable digital circuit capable of generating a variable frequency from $74.2 \mathrm{kHz}$ to $130 \mathrm{kHz}$. The amplifier consisted of a MOSFET driver (ST Microelectronics L6384) and two high-power N-channel MOSFETs (Fairchild FQP19N20C). The output of the amplifier stage produced a square wave. A filter blocked the high frequency components of the square wave so that only a sinusoidal wave could reach the work resonator. A filter consisted of a hand wound inductor L1 and a capacitor C1 (Kemet R463N333000N0M) placed in series. The work resonator was also constructed from a capacitor (Kemet R75QN3100AA00K) and an inductor, but instead they were placed in parallel. The work coil L2 was a hand turned copper tube. Both C1 and C2 consisted of many of the specified capacitors paced in parallel to achieve the required capacitance. Placement in parallel allowed the 
whole circuit to operate at an increased power level than any individual capacitor was capable of handling (Figure 2).

The test samples were placed in the center of the coil for exposure to the magnetic field. While the AMF generator could be operated at a lower power by setting the input frequency higher, it was capable and primarily used at a frequency of $74.2 \mathrm{kHz}$ which produced a magnetic field with strength of $10 \mathrm{kA} / \mathrm{m}(12.6 \mathrm{mT})$.

\subsection{Bulk heating of SPIONs}

A series of bulk heating experiments were performed to test whether the machine was capable of generating heat via magnetic activation of the nanoparticles. For this kinetic study, readily available SPIONs (nanoComposix, San Diego, CA) at a concentration of $20 \mathrm{mg} / \mathrm{ml}$ were used. The SPIONs-solution was placed in the work coil and gradually exposed to AMF for $0.5,1.0,1.5,2.0,2.5$, $3.0,3.5,4.0,5,6,7,8,10,15,20,25$, and 30 minutes to establish the minimum time required for the maximum temperature rise. The initial and final temperature was measured using a thermocouple. To verify that bulk heating was caused from the interaction specifically with ferric oxide nanoparticles, four different control samples were studied including deionized (DI) water, 1X phosphate buffered saline (PBS) solution, tap water and gold nanoparticles (nanoComposix, San Diego, CA) at the same concentration as SPIONs. Based on the above experiments the optimal heating time of 4 minutes was used to determine the dose-dependence of the effect using varying concentrations; $2.5,5,10,15$, and 20 $\mathrm{mg} / \mathrm{ml}$ of SPIONs. A volume of $400 \mu \mathrm{l}$ was used for all treatment samples. Each type of experiment was performed twice, the data were the average over the two experiments and plotted with the standard error of the mean value.

\subsection{HA-DESPION binding to CD44}

UT-SCC-14 cells were plated in T-25 $\mathrm{cm}^{2}$ flasks in complete growth media at $5 \times 10^{5}$ cells/flask for 2 days and allowed to attach overnight. Three different experimental groups were established: 1) control cells, 2) cells treated with FITC-DESPIONs, and 3) cells treated with FITC-HA-DESPIONs. Next day media of control cells were replaced with prewarmed media while group 2 and 3 cells were treated with FITC-DESPIONs and FITC-HA-DESPIONs at $200 \mu \mathrm{g} / \mathrm{ml}$ respectively. Cells were allowed to grow for 24 hours in an incubator at $37^{\circ} \mathrm{C}$ with $5 \% \mathrm{CO}_{2} / 95 \%$ air. After 24 hours of incubation, cells were washed four times with pre-warmed growth media, and trypsinized from flasks. Freshly trypsinized cells were centrifuged at $1100 \mathrm{rpm}$ for 5 minutes, washed twice with PBS solution, and resuspended at $1 \times$ $10^{6}$ cells $/ \mathrm{ml}$ in PBS solution. Cells were, then, immediately run on a FACS Canto II flow cytometer (BD Biosciences) to measure the fluorescence (FITC) attached to the cells.

\subsection{Cell culture for AMF treatment}

UT-SCC-14 cells were plated in $35 \mathrm{~mm}^{2}$ dishes in complete growth media at $2 \times 10^{5}$ cells/dish for 3 days and allowed to grow in an incubator at $37^{\circ} \mathrm{C}$ with $5 \%$ $\mathrm{CO}_{2} / 95 \%$ air. Three different experimental groups were established: 1) control cells, 2) cells treated with HA-DESPIONs, and 3) cells treated with DESPIONs. Each treatment group was replicated to compare treatment effects in the presence and absence of AMF. After overnight incubation, cells were treated with HA-DESPIONs/DESPIONs at $200 \mu \mathrm{g} / \mathrm{ml}$ for 24 hours. After 24 hours of incubation with nanoparticles, cells were washed four times with pre-warmed growth media, and resuspended in complete growth media. Cells were then exposed to AMF-induced hyperthermia for 30 minutes at room temperature. Cells were incubated at $37^{\circ} \mathrm{C}$ for 24 hours before the analysis of apoptotic cell death by flow cytometry.

\subsection{Flow cytometry analysis after AMF treatment}

To assess the effects of treatments, cells were trypsinized 24 hours post-treatment. Freshly trypsinized cells were centrifuged at $1100 \mathrm{rpm}$ for 5 minutes, washed twice with PBS solution, and resuspended at $1 \times 10^{6}$ cells $/ \mathrm{ml}$ in PBS solution. Cells were then dual stained for CD44 (FITC) at $8 \mu \mathrm{l} / \mathrm{ml}$ and Annexin V (PE) at $8 \mu \mathrm{l} / \mathrm{ml}$ (BD Biosciences) to evaluate the percentage of cells undergoing apoptosis in the CD44 expressing population. Each sample was stained for 1) cells only, 2) isotype-stained cells, 3) CD44-stained cells, 4) Annexin-stained cells 5) CD44/Annexin dual staining. Cells were covered with aluminum foil, and incubated at $4^{\circ} \mathrm{C}$ for 35 minutes, protected from light. After staining, cells were further washed twice with cold PBS, and resuspended in $1 \mathrm{ml}$ of PBS solution. Cells were, then, immediately run on a FACS Canto II flow cytometer (BD Biosciences) to evaluate the percentage of cells undergoing apoptosis in the CD44 expressing population using appropriate regions of interest.

\section{Results}

\subsection{Bulk heating results}

The initial studies verified that the AMF generator was capable of activating the magnetic nanoparticles to produce significant increases in temperature. Figure 3 shows that immediately after the magnetic field is initiated, the magnetic nanoparticles $(20 \mathrm{mg} / \mathrm{ml})$ became activated and, as a consequence, the heating effect was observed in the form of a rise in temperature. The minimum time of activation to achieve the maximum rise in temperature in the test scenario was approximately 4 minutes (Figure 3 ). At 4 minutes of activation, the temperature was recorded as $37.3^{\circ} \mathrm{C}$ representing a $12.5^{\circ} \mathrm{C}$ rise from the initial room temperature. Further increase in time did not show a significant change in temperature indicating that the temperature rise plateaued when the heating of the 
magnetic nanoparticles reached a point of maximum activation (Figure 3).

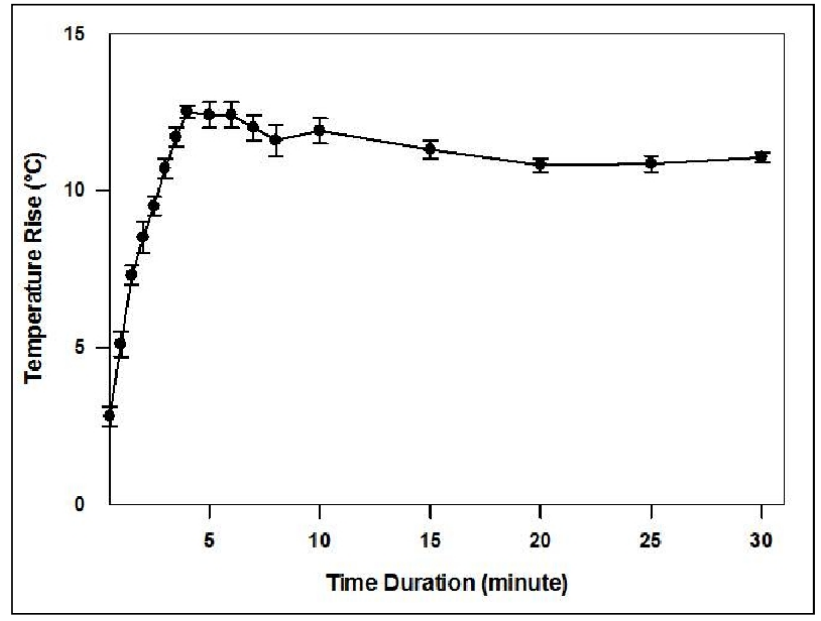

Figure 3: Generation of heat as a function of magnetic field duration; temperature changes plateau at longer AMF activation periods in $20 \mathrm{mg} / \mathrm{ml}$ of SPION solution.

Table 1: Initial (at 0 minute), and final (at 4 minutes) temperatures obtained from a series of control solutions.

\begin{tabular}{ccccc}
\hline \hline Time & \multicolumn{4}{c}{ Temperature $\left({ }^{\circ} \mathrm{C}\right)$} \\
\cline { 2 - 5 } (minute) & DI water & PBS solution & Gold NPs & SPIONs \\
0 & 24.7 & 24.5 & 24.5 & 24.8 \\
4 & 24.6 & 24.3 & 24.6 & 37.6 \\
\hline \hline
\end{tabular}

Table 1 shows that none of the control samples, DI water, PBS solution and gold nanoparticles produced a significant rise in their final temperatures in comparison to the SPIONs.

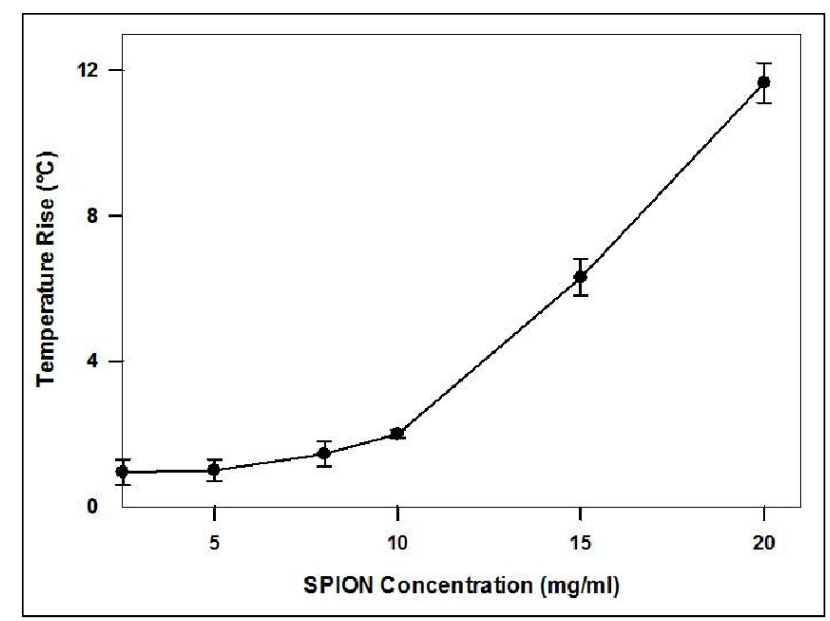

Figure 4: The effect of SPION concentration on temperature rise.

In Figure 4, the dose-dependence studies show that there was a relationship between the concentration of SPIONs and the amount of bulk heating achieved through the exposure to the AMF. As expected the largest rise in the temperature of $11.6^{\circ} \mathrm{C}$ above the initial room temperature $\left(24.8^{\circ} \mathrm{C}\right)$ was observed at 4 minutes of exposure to the AMF with the highest concentration tested $(20 \mathrm{mg} / \mathrm{ml})$. There appeared to be very little effect up to a concentration of $10 \mathrm{mg} / \mathrm{ml}$ after which there was linear correlation between SPIONs concentration and temperature rise.

\subsection{HA-DESPIONs binding test results}

After 24 hours of incubation, cells treated with FITCHA-DESPIONs showed significant amount of fluorescence attached to the cells (Figure 5C) compared to the FITC-DESPIONs-treated cells (Figure 5B), and control cells (Figure 5A). The mean fluorescence intensity measured in control, FITC-DESPIONs, and FITC-HA-DESPIONs treated cells were $320 \pm 24,427 \pm 35$, and $17292 \pm 457$ respectively.

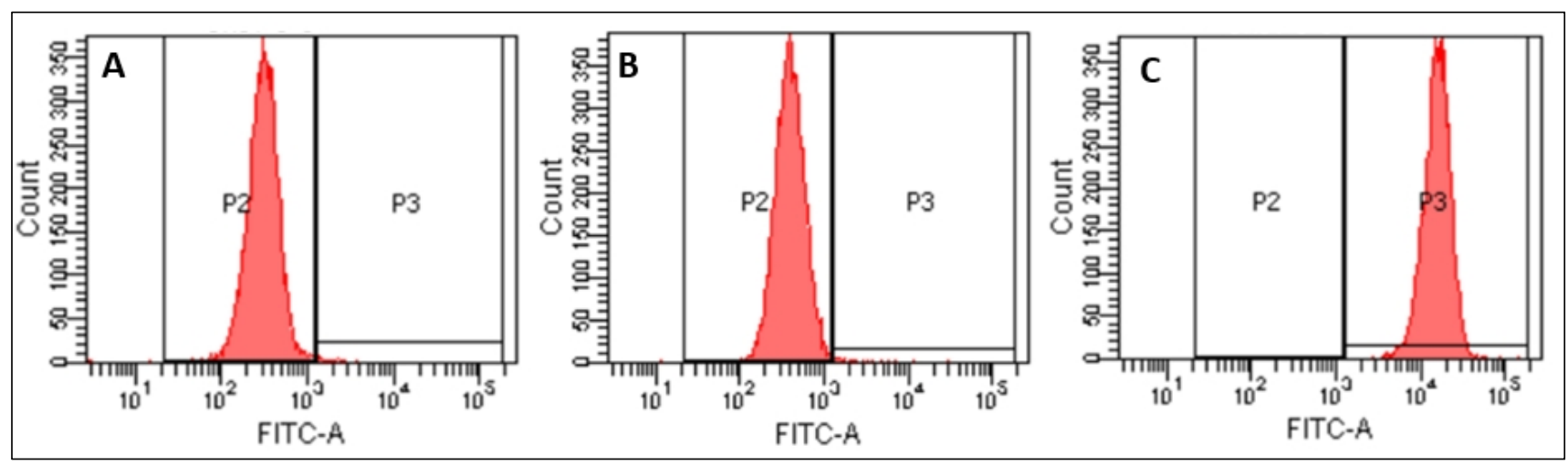

Figure 5: Plots A, B, and C are the representative flow cytometric plots demonstrating amount of fluorescence (FITC) attached in control, DESPION-treated, and HA-DESPION-treated cells respectively. 


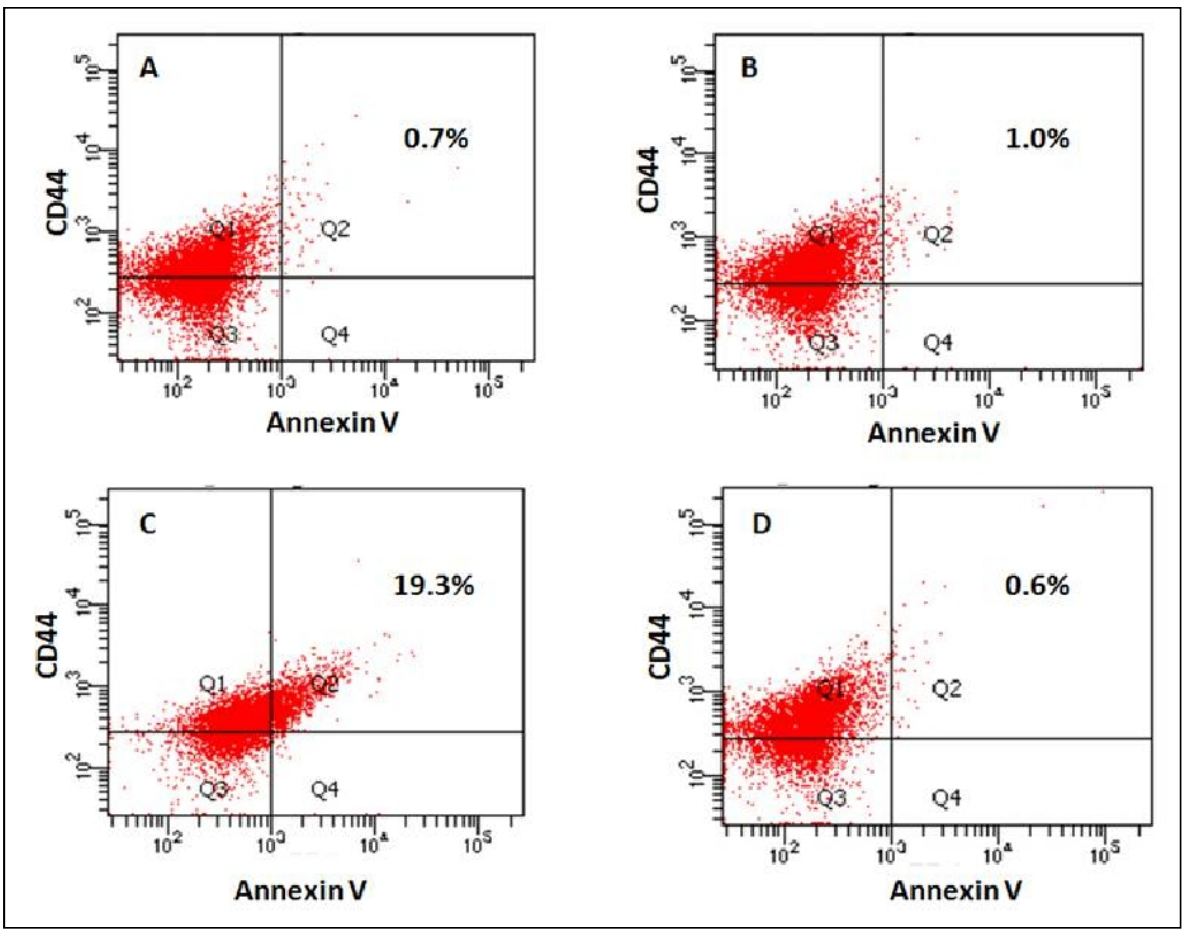

Figure 6: Figures A, B, and C are the representative flow cytometric plots demonstrating \% of apoptotic CD44 cell death detected in control cells, DESPION $(200 \mu \mathrm{g} / \mathrm{ml})$ treated cells, and HA-DESPION $(200 \mu \mathrm{g} / \mathrm{ml})$ treated cells when the AMF is turned on while figure D is the representative HA-DESPION treated cells when the AMF is turned off.

\subsection{AMF treatment (flow cytometric analysis) results}

Following exposure to AMF, non-conjugated DESPIONs were unable to induce targeted hyperthermia and hence had no effect on CD44 cell death as they were not attached to the cells (Figure 6B), similar to control cells (Figure 6A). However, there was a significant amount of cell death (19.3\%) in CD44 expressing cells treated with HA-DESPIONs and exposed to AMF (Figure 6C). When the magnetic field was turned off, HA-DESPION treated cells had no hyperthermic effect on the CD44 population (Figure 6D), similar to all treatment groups (figures not shown here).

\section{Discussion}

In HNSCCs, HA-CD44 interaction plays a significant role in tumor progression and metastasis. As a result, HA functionalized nanomaterials have been investigated to target CD44 populations and to selectively deliver drugs for the treatment. ${ }^{25,26}$ The dextran coated nanoparticles (HA-DESPIONs) used in this study have been developed by a co-precipitation method followed by post synthetic functionalization of $\mathrm{HA}^{22}$ These nanoparticles have previously been shown to be detectable in tumors and atherosclerotic plaques using static magnetic fields to provide contrast for magnetic resonance imaging. ${ }^{19,20,22}$ In addition, their cellular uptake and binding to CD44-overexpressed cells have also been tested. This indicated the importance of cell specificity and targeting in relation to hyperthermia therapy. ${ }^{27}$
As mentioned previously, another potential application of nanoparticles based on their ferric oxide content is their ability to produce heat in response to AMF. For this study we have generated a simple and inexpensive in-house fabricated AMF generator. The apparatus was able to generate heat energy via magnetic activation of magnetic nanoparticles. However, this required a high concentration of nanoparticles. Using a high concentration of nanoparticles in biomedical applications can have serious side effects owing to the properties of dose-dependent toxicity. ${ }^{28}$ In addition, high concentrations of magnetic nanoparticles dominates the signal intensity of T2-weighted MRI when they are used as contrast enhancer. ${ }^{29}$ To address these limitations, we used comparatively a low dose (200 $\mu \mathrm{g} / \mathrm{ml}$ ) of HA-DESIPONs for the cellular study, and provided cells sufficient ( 24 hours) time to interact with the nanoparticles, and exposed cells to the AMF for the maximum time of 30 minutes according to the bulk heating kinetic study. 30 minutes was selected for the cellular studies since, generally, the internalization of nanoparticles within cells is likely to occur in a time-dependent manner and after a certain threshold is reached the uptake is expected to plateau when cells have reached a point of maximum saturation. Similarly, cell viability subsequent to nanoparticle uptake is expected to be either unaffected or decrease as a function of time. ${ }^{28}$ The cytotoxic property of HA-DESPIONs was previously tested using a growth inhibition assay in which UT-SCC-14 cells did not show 
any cytotoxic effect with the treatment of HA-DESPIONs at the concentration of $200 \mu \mathrm{g} / \mathrm{ml}$ for up to 48 hours. ${ }^{18}$

In one recent study, magnetically induced hyperthermia was achieved using SPIONs in a head and neck cancer xenograft model. ${ }^{12}$ However, this was an untargeted approach and the interactions of a targeted approach using HA-DESPIONs directed at CD44, in the presence of an AMF, have not been previously investigated. In terms of the in vitro studies, our results suggested that HA-DESPIONs bind to CD44 expressing cells but required 24 hours of incubation to result in a significant amount of apoptotic CD44 cell death as hyperthermia treatments performed immediately after the addition of nanoparticle solution had no significant difference in apoptotic cell death. The data in Figure 6C clearly show the apoptotic population emanating from the CD44-postive cells and not the CD44-negative population. DESPIONs, without HA conjugation, did not show any effects on either the CD44 positive or negative cells (Figure 6B). Our result is consistent with Kamat et al. ${ }^{22}$ According to their study, HA receptor (CD44) expressed on the tumor cells can be recognized by HA-DESPIONs. The enzyme linked immunosorbent assay (ELISA) demonstrated the binding of HA-DESPIONs, while DESPIONs could not. Further, their cellular studies showed HA-DESPIONs effectively inhibited the binding between the HA polymer and CD44 while the DESPIONs without HA did not have any effect on HA-CD44 interaction.

The data presented in this study are encouraging but must be considered as preliminary and the present study does have limitations. First, we need to examine other HNSCC cell lines with differing expression levels of CD44 and hyperthermic properties as described in a previous publication. ${ }^{18}$ The studies in this report were performed at room temperature rather than body temperature due to the limitations of the current apparatus; we are developing a system that can be situated in a $37^{\circ} \mathrm{C}$ incubator. Several associated studies are ongoing. We have preliminary data that intravenous injection of HA-DESPIONS into nude mice bearing UT-SCC-14 xenografts can be detected in a 3.0 Tesla benchtop MRI and that changes in MRI signal caused by radiation treatment correlate with observed changes in CD44 expression measured by immunohistochemistry and gene expression. ${ }^{30}$ In addition we are exploring an $\mathrm{RF}$ pulse sequence technique to enable activation of hyperthermia of HA-DESPIONS in a clinical MRI. ${ }^{31}$

\section{Conclusion}

In this study we have demonstrated that a simple AMF generator was able to activate magnetic nanoparticles and a dose-dependent heating effect was obtained. The flow cytometry results demonstrated that HA-DESPIONs were targeted to UT-SCC-14 cells through binding to the overexpressed surface receptor CD44 and were activated in the presence of an AMF to generate a significant amount of localized heat that ultimately led to tumor cell death. This preliminary study needs to be further elaborated at different time intervals and expanded to other HNSCC cell lines but the early results suggest a promising strategy to specifically target CSCs in HNSCCs for treatment.

\section{Conflict of interest}

The authors declare that they have no conflicts of interest. The authors alone are responsible for the content and writing of the paper.

\section{Acknowledgement}

This work is funded by Department of Radiation Oncology, Beaumont Health System, and Oakland University Provost Graduate Student Research Award.

\section{References}

1. Vermorken JB, Remenar E, van Herpen C, et al. Cisplatin, fluorouracil, and docetaxel in unresectable head and neck cancer. N Engl J Med. 2007;357(17):1695-704.

2. Pignon JP, le Maître A, Maillard E, et al. Meta-analysis of chemotherapy in head and neck cancer (MACH-NC): an update on 93 randomised trials and 17,346 patients. Radiother Oncol. 2009;92(1):4-14.

3. Reya T, Morrison SJ, Clarke MF, Weissman IL. Stem cells, cancer, and cancer stem cells. Nature. 2001;414(6859):105-11.

4. Prince ME, Sivanandan R, Kaczorowski A, et al. Identification of a subpopulation of cells with cancer stem cell properties in head and neck squamous cell carcinoma. Proc Natl Acad Sci U S A. 2007;104(3):973-8.

5. Wang SJ, Wong G, de Heer AM, et al. CD44 Variant isoforms in head and neck squamous cell carcinoma progression. Laryngoscope. 2009;119(8):1518-30.

6. Toole BP, Slomiany MG. Hyaluronan, CD44 and Emmprin: partners in cancer cell chemoresistance. Drug Resist Updat. 2008;11(3):110-21.

7. Laurent TC, Laurent UB, Fraser JR. The structure and function of hyaluronan: An overview. Immunol Cell Biol. 1996;74(2):A1-7.

8. Bartolazzi A, Peach R, Aruffo A, Stamenkovic I. Interaction betwenn CD44 and hyaluronate is directly implicated in the regulation of tumor-development. J Exp Med. 1994; 180(1):53-66.

9. Liu W, Frank JA. Detection and quantification of magnetically labeled cells by cellular MRI. Eur J Radiol. 2009;70(2):258-64.

10. Dixon WT, Blezek DJ, Lowery LA, et al. Estimating amounts of iron oxide from gradient 
echo images. Magn Reson Med. 2009; 61(5):1132-6.

11. Ang KK, Andratschke NH, Milas L. Epidermal growth factor receptor and response of head-and-neck carcinoma to therapy. Int J Radiat Oncol Biol Phys. 2004;58(3):959-65.

12. Zhao Q, Wang LN, Cheng $\mathrm{R}$, et al. Magnetic nanoparticle-based hyperthermia for head \& neck cancer in mouse models. Theranostics. 2012;2(1):113-21.

13. Drake P, Cho H-J, Shih P-S, et al. Gd-doped iron-oxide nanoparticles for tumour therapy via magnetic field hyperthermia. J Mater Chem. 2007;17(46):4914-8.

14. Smolkova IS, Kazantseva NE, Babayan V, et al. Alternating magnetic field energy absorption in the dispersion of iron oxide nanoparticles in a viscous medium. J Magn Magn Mater. 2015;374:508-15.

15. Thomas LA, Dekker L, Kallumadil M, et al. Carboxylic acid-stabilised iron oxide nanoparticles for use in magnetic hyperthermia. J Mater Chem. 2009; 19(36):6529-35.

16. Muller S. Magnetic fluid hyperthermia therapy for malignant brain tumors-an ethical discussion. Nanomedicine. 2009;5(4):387-93.

17. Provenzale JM, Silva GA. Uses of Nanoparticles for Central Nervous System Imaging and Therapy. AJNR Am J Neuroradiol. 2009; 30(7):1293-301.

18. Thapa R, Galoforo S, Kandel SM, et al. Radiosensitizing and hyperthermic properties of hyaluronan conjugated, dextran-coated ferric oxide nanoparticles: Implications for cancer stem cell therapy. J Nanomater. 2015; 2015:840594.

19. El-Dakdouki MH, Zhu DC, El-Boubbou K, et al. Development of multifunctional hyaluronan-coated nanoparticles for imaging and drug delivery to cancer cells. Biomacromolecules. 2012;13(4):1144-51.

20. El-Dakdouki MH, El-Boubbou K, Kamat M, et al. CD44 Targeting magnetic glyconanoparticles for atherosclerotic plaque imaging. Pharm Res. 2014;31(6):1426-37.

21. Wilson GD, Marples B, Galoforo S, et al. Isolation and genomic characterization of stem cells in head and neck cancer. Head Neck. 2013; 35(11):1573-82.

22. Kamat M, El-Boubbou K, Zhu DC, et al. Hyaluronic acid immobilized magnetic nanoparticles for active targeting and imaging of macrophages. Bioconjug Chem. 2010; 21(11):2128-35.

23. El-Boubbou K, Zhu DC, Vasileiou C, et al. Magnetic glyco-nanoparticles: a tool to detect, differentiate, and unlock the glyco-codes of cancer via magnetic resonance imaging. J Am Chem Soc. 2010;132(12):4490-9.

24. El-Dakdouki MH, El-Boubbou K, Zhu DC, Huang $\mathrm{X}$. A simple method for the synthesis of hyaluronic acid coated magnetic nanoparticles for highly efficient cell labelling and in vivo imaging. Rsc Adv. 2011;1(8):1449-52.

25. Thapa R, Wilson GD. Head and neck cancer: Current treatment options and associated challenges. Sch J App Med Sci. 2016; 4(2D):590-600.

26. Thapa R, Wilson GD. The importance of CD44 as a stem cell biomarker and therapeutic target in cancer. Stem Cells Int. 2016;2016:2087204.

27. Thomas RG, Moon MJ, Lee H, et al. Hyaluronic acid conjugated superparamagnetic iron oxide nanoparticle for cancer diagnosis and hyperthermia therapy. Carbohydr Polym. 2015;131:439-46.

28. Singh N, Jenkins GJS, Asadi R, Doak SH. Potential toxicity of superparamagnetic iron oxide nanoparticles (SPION). Nano Rev. 2010;1.

29. Wabler M, Zhu W, Hedayati M, et al. Magnetic resonance imaging contrast of iron oxide nanoparticles developed for hyperthermia is dominated by iron content. Int J Hyperthermia. 2014;30(3):192-200.

30. Wilson GD, Thibodeau BJ, Fortier LE, et al. Cancer Stem Cell Signaling during Repopulation in Head and Neck Cancer. Stem Cells Int. 2016;2016:1894782.

31. Yee S, Ionascu D, Thapa R, Wilson GD. An exploratory RF pulse sequence technique used to induce differential heating in tissues containing iron oxide nanoparticles for a possible hyperthermic adjuvant effect to radiotherapy. Med Phys. 2014;41:356. 